\title{
Pathological studies on fallopian tube in relation to uterine lesions and ovarian abnormalities
}

\author{
E. A. Mahdy \\ Department of Pathology, Faculty of Veterinary Medicine, Beni-Suef University, Beni-Suef, Egypt.
}

\begin{abstract}
A total number of 50 genital tracts were collected from cows slaughtered at "Belefia" abattoir in "Beni-Suef" governorate, Egypt. The genitalia were inspected grossly and the ovarian activity was noticed. Tissue specimens were taken from the tips of uterine horns, fallopian tubes, ovaries of both sides. The fallopian tubes were cut and sampled at three levels namely, infundibulum, ampulla, isthmus. All tissue specimens were fixed in $\mathbf{1 0 \%}$ formalin solution, embedded in paraffin wax, sectioned at $5 \mu \mathrm{m}$ and stained with hematoxylin and eosin stain (Bancroft and Stevens 1996) and examined microscopically. The pathological changes in the fallopian tube in relation to lesions in the uterus and ovarian activity were investigated. The uterine pathological lesions were endometritis (12\%), adenomyosis (12\%), and cystic glandular hyperplasis $(8 \%)$. Inflammation of the fallopian tube salpingitis, was graduated as mild degree(18\%), moderate degree(2\%), and severe degree $(2 \%)$. The intraepithelial microcysts of the uterine tube represented $8 \%$ of the examined cases.
\end{abstract}

The tubular female genitalia arised from the embryonic paramesonephric (MÜllerian) duct (Noden and de lahunta, 1985) and are responsible for capture, transport, and sustenance of the ovum and conceptus. Within baboon oviduct (fallopian tube) ampulla, estradiol induced hypertrophy, hyperplasia, ciliogenesis and secretory activity whereas adding progesterone to the treatment regimen resulted in atrophy, deciliation, apoptosis, and loss of secretory activity (Verhage et al., 1990). The uterine tube consists of three major segments; the infundibulum, ampulla and isthmus, in that order from the ovarian end to the uterus. Abe and Oikawaa, (1993) showed that percentage of ciliated cells significantly decreased in the fimbriae and ampulla in the luteal phase, but not in the other regions. The height of ciliated cells decreased dramatically in the fimbriae, ampulla, ampullar-isthmic junction, isthmus, and uterotubular junction at the luteal phase, not in the fimbriae and ampullae. As reported by McEntee, (1990), the major macroscopic abnormalities in this tubular organ are cysts, accumulation of fluid in the lumen with or without accompanying inflammation, and neoplasia. Neoplasms of this area are extremely rare in domestic animals (McEntee, 1990); women (Hue et al., 1950). As regards incidence of salpingeal lesions, the only agreement is that they are much more common than the diagnosis of them. They are recognized to be important in the cow and sow, but not in

\footnotetext{
* Corresponding author. Tel.: +20 082 2322066;

Fax: +20 0822327982

E-mail address: mahdy@bsu.edu.eg

(Emad A. Mahdy)
}

other species (Jubb et al., 1993). In the present study, pathological changes in the fallopian tube in relation to lesions in the uterus and ovarian activity were investigated.

\section{Material and methods}

A total number of 50 genital tracts were collected from cows slaughtered at "Belefia" abattoir in "Beni-Suef" governorate, Egypt. The genitalia were inspected grossly and the ovarian activity was noticed. Tissue specimens were taken from the tips of uterine horns, fallopian tubes, ovaries of both sides. The fallopian tubes were cut and sampled at three levels namely, infundibulum, ampulla, and isthmus. All tissue specimens were fixed in $10 \%$ formalin solution, embedded in paraffin wax, sectioned at 5 um and stained with hematoxylin and eosin stain (Bancroft and Stevens, 1996) and examined microscopically.

\section{Results}

Histologically, the wall of the the fallopian tube of cattle consisted of three layers, namely, mucosa (endosalpinx), intermediate muscle layer (myosalpinx) and an outer serosa. The mucosa is thrown into longitudinal folds, called primary folds, increasing in number toward the fimbria and were lined by three types of epithelial cells, ciliated columnar epithelial cells, secretory cells and basal cells. The number of ciliated cells increased gradually from the isthmus towards the infundibulum. The mucosa of ampullary and infundibular regions showed secondary folds. The myosalpix consisted of an inner circular and an outer longitudinal layer. The lumen of the fallopian tube was narrow in the isthmus, wide in 
the ampulla and moderately wide in the infundibulum. This lumen is almost clear, contained no mucous or exudate in normal animals. In inflammed organ, cellular exudate consisting of infiltrating leucocytes and desquamated epithelial cells were seen. The types of leucocytes depend on the type of inflammation; the cells were neutrophils in suppurative inflammation and mononuclear cells in acute and chronic non-suppurative inflammation.

Macroscopically, fallopian tube lesions could not be detected. Microscopically, in the present study, inflammatory changes of endometrium was found in 6/50 cases (12\%); all of these cases were associated with variable degrees of salpingitis at the same time except one case. Macroscopically, yellowish green colored flakes of pus were accumulated in the uterine lumen of a case with purulent endometritis Endometritis was classified as: chronic suppurative endometritis in 5 cases and one case of eosinophilic endometritis. Histopathological changes consisted of periglandular and subepithelial accumulations of neutrophils and lymphocytes with migration of neutrophils into the glandular and uterine lumens (Fig. 1). In more advanced chronic cases, the uterine glands showed atrophy, degeneration and cystic dilatation. In case of eosinophilic endometritis, the histopathological lesions consisted of submucosal oedema, diffuse and focal eosinophilic infiltrations and destruction of the endometrial surface epithelium at many areas (Fig.2). The same case of eosinophilic endometritis showed unilateral lymphocytic salpingitis of moderate degree.

Adenomyosis was observed in the uterus of $6 / 50(12 \%)$ of the examined cases. Three cases of them were associated with mild salpingitis. Only one presented both chronic suppurative endometritis and granulosa cell tumor of the ovary (Fig.3). In these cases, the endometrial glands and stroma were embedded in-between the myometrium muscle fibers (Fig.4). In one of these cases, the oviduct showed active proliferation of the surface epithelium mostly indicating hormonal influence (Fig.5). The stroma reacted similar to uterine endometrial stroma. The isthmus was less severely affected than infundibulum. In other adenomyosis cases, the fallopian tube epithelial lining showed proliferative activity, and intraepithelial microcysts. The lamina propria showed chronic inflammatory changes (Fig.6). In another case, the nuclei of non-ciliated epithelial lining were more rounded than elliptical and multilayered with sparse leucocytic infiltration in the lamina propria. The active secretory cells appeared disperse (Fig.7). In some cases, the fallopian tube showed submucosal edema, congestion and degenerated epithelial lining (Fig.8).

In the uterus, cystic glandular hyperplasia cases represented $8 \%$, most of them were associated with both endometritis and salpingitis. One case was associated with granulose cell tumor. The changes were diffuse; the covering epithelium appeared secretory active, the glands were distended and most glandular epithelium appeared flattened or cuboidal (Fig.9). The fallopian tube fimbriae of such cases showed multiple mucosal folds, edema, and congestion and secretory active epithelial cells. The cells were columnar with vesicular nuclei (Fig.10). In the isthmus, there were low mucosal folds, fold adhesion and columnar epithelial cells with centerally located nuclei.

Generally, abnormalities in the fallopian tube represented about $24 \%$ of the microscopically examined cases. Salpingitis was common, mostly bilateral and was classified as acute of mild degree (18\%), in which there was slight mononuclear cell infiltration of the mucosa and congested mucosal blood vesssels in lamina propria and loss of epithelial cilia (Fig.11), moderate degree $(2 \%)$ in which there was thickening of mucosal folds by massive cellular infiltration and edema, with destruction of the epithelium at many parts (Fig.12), or severe degree $(2 \%)$ in which the mucosal folds were fused, destroyed at great areas and replaced by cellular infiltrations with obliteration of the lumen (Fig.13).

Three out of 11 cases with salpingitis showed adenomyosis at the same time while only one case showed both suppurative endometritis and granulosa cell tumor. Seven cases out of 11 cases with salpingitis $(60.33 \%)$ were associated with endometritis at the same time. Four cases out of 5 granulosa cell tumor cases showed mild degree of salpingitis.

Intraepithelial cysts in the uterine tube were detected microscopically in $8 \%(4 / 50)$ of the examined cases, and three cases of them were associated with salpingitis of mild degree. The cysts were abundant and lined at least partially by cuboidal to columnar epithelium in the mucosa of the infundibulum or ampulla (Fig.14). $50 \%$ of the intraepithelial cysts cases showed adenomyosis. 

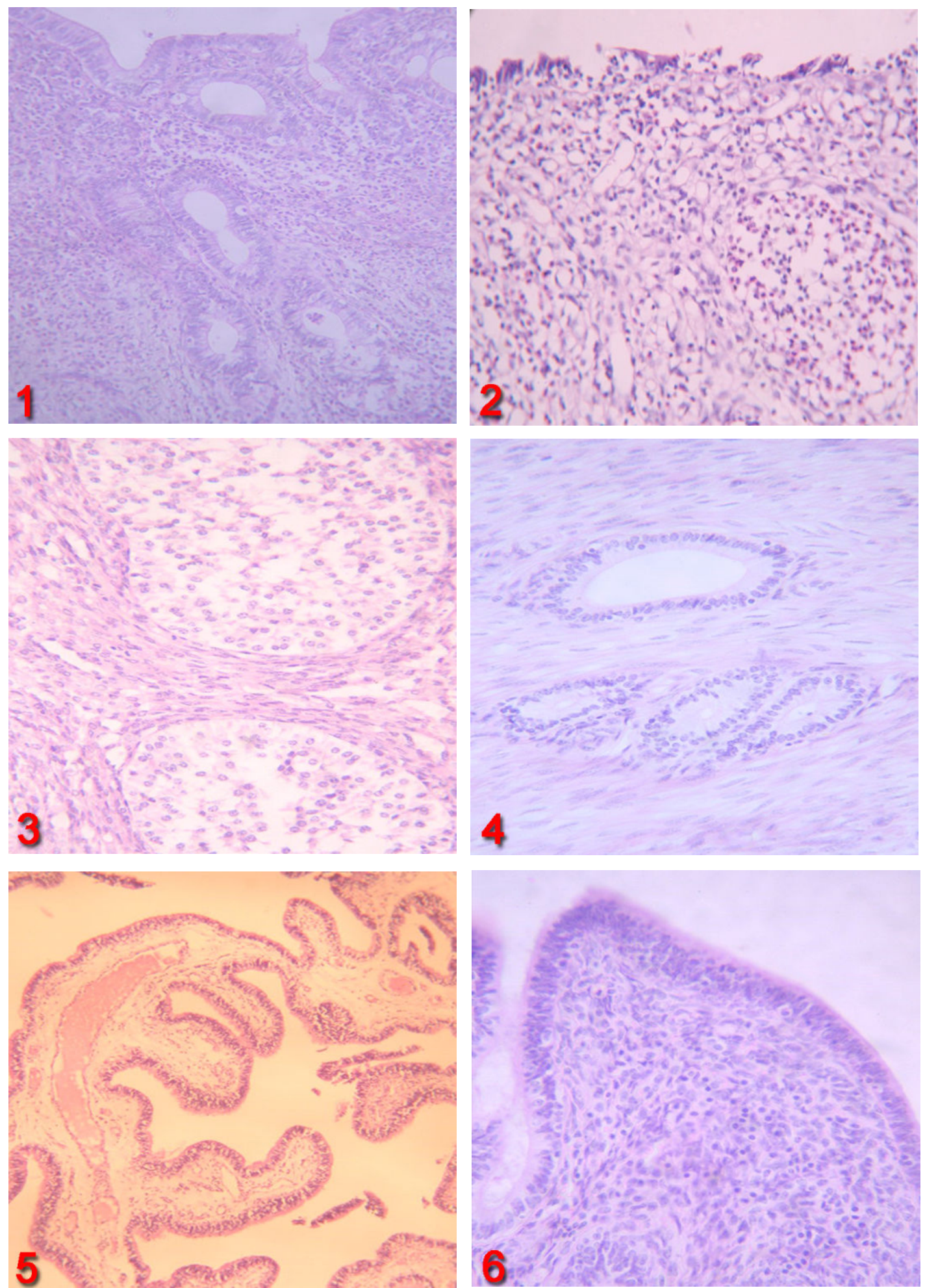

Fig.1: Uterus;chronic suppurative endometritis.Notice the subepithelial infiltrations of neutrophils and lymphocytes (H\&E-X200).

Fig.2: Uterus; eosinophilic endometritisin which there is submucosal infiltration of eosinophils and destruction of endometrial surface epithelium infiltrations of neutrophils and lymphocytes (H\&E-X400).

Fig.3: Ovary; granulose cell tumor (H\&E-X400).

Fig.4: Uterus; the endometrial glands are embedded in between the muscle fibers (adenomyosis) (H\&E-X400).

Fig.5: Uterine tube: Notice the active proliferation of the surface epithelium (H\&E-X100).

Fig.6: Uterine tube: The lamina propria showed chronic inflammatory change (H\&E-X400). 

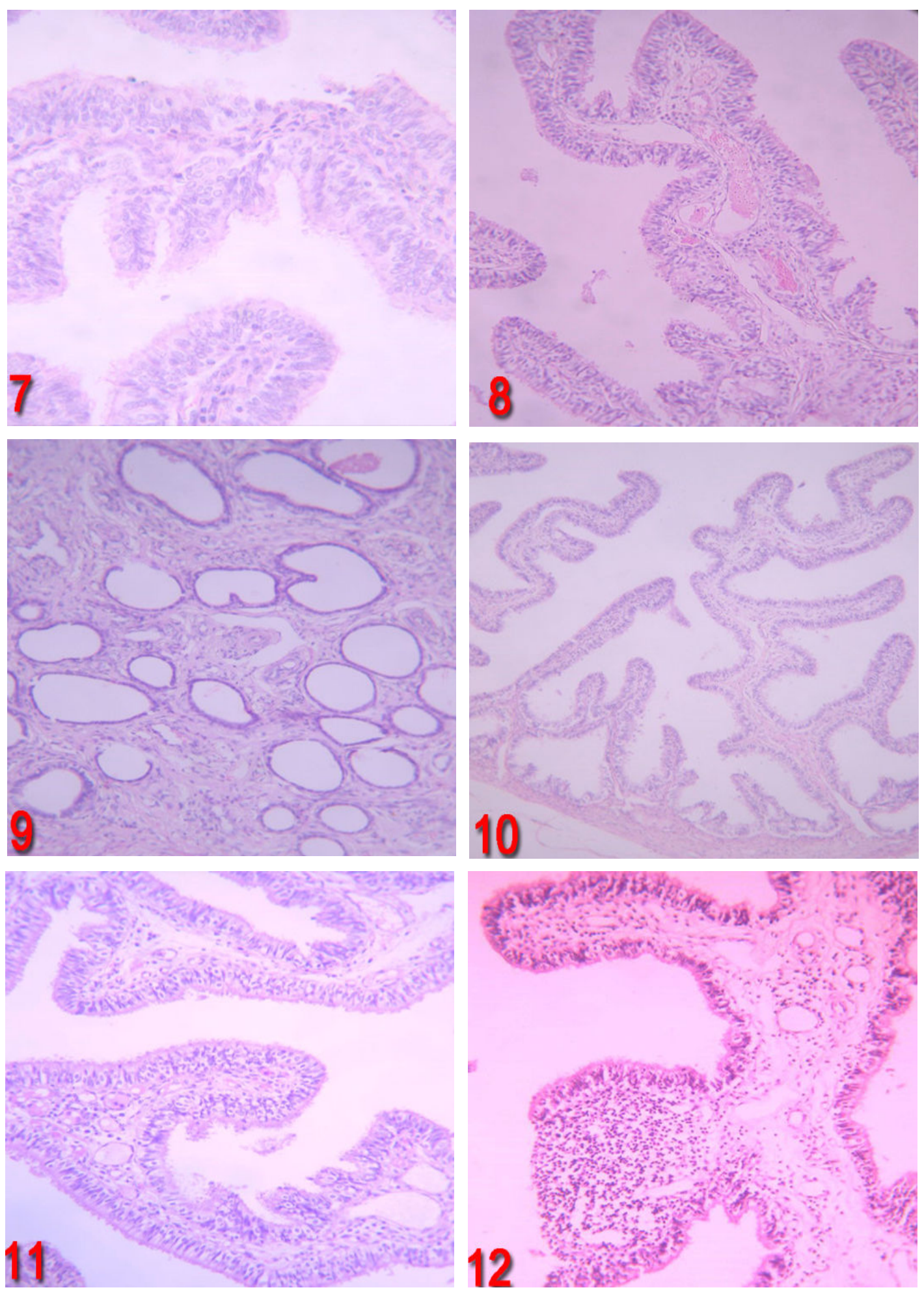

Fig.7: Uterine tube: Notice the multilayered nonciliated rounded epithelial lining cells (H\&E-X400).

Fig.8: Uterine tube: Submucosal edema and congestion (H\&E-X200).

Fig.9: Uterus; Cystic glandular hyperplasia. Notice the cystically dilated, uterine glands with flattened epithelial lining (H\&E-X200).

Fig.10: Fallopian tube showed multiple mucosal folds, edema and congestion (H\&E-X100).

Fig.11: Fallopian tube showed slight mononuclear cell infiltration and congestion of the blood vessels in the lamina propria(H\&E-X200).

Fig.12: Fallopian tube showed thickening of the mucosal folds by massive cellular infiltration (H\&E-X200). 


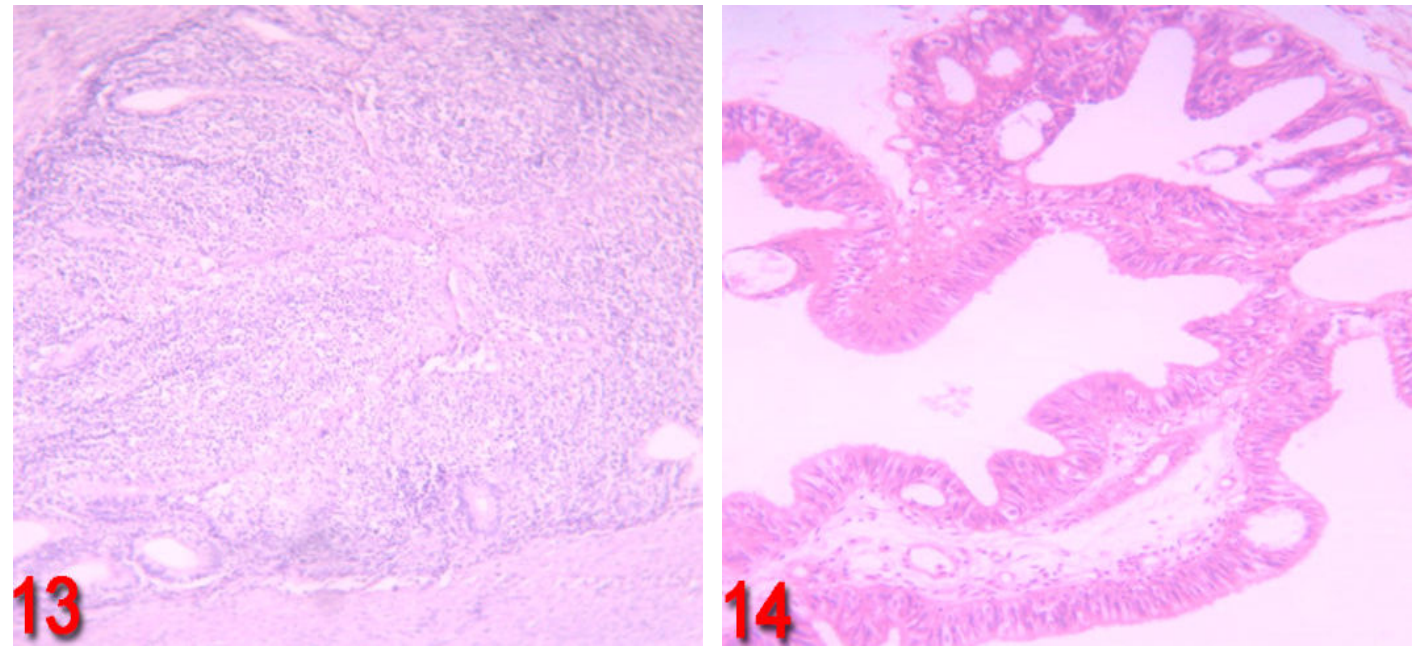

Fig.13: Fallopian tube showed severe degree of salpingitis (the mucosal folds are fused and destroyed and replaced by cellular infiltration which obliterate the lumen) (H\&E X100).

Fig.14: Fallopian tube showed intraepithelial mucosal microcysts (H\&E-X100).

\section{Discussion}

In the present study, the incidence of endometritis was relatively high (12\%) and was associated in most cases with ascending salpingitis. The macroscopic and microscopic findings of endometritis were identical to previously mentioned by (Jubb et al., 1993; Karadase and Timurkaan, 2001; Hatipoglu et al., 2002). Doig et al., (1980) found that endometritis and salpingitis following uterine inoculation with some strains of ureaplasma were pathogenic for the upper reproductive tract of the cow. McDougall, (2005) described the gross pathology, bacteriology and histopathology of the reproductive tracts of dairy cows that failed to conceive or maintain pregnancy and found that $36 \quad(34 \%)$ of cows had one or more gross lesions which involved the ovary, uterine tube (fallopian tube), uterus or vagina. In only five cows were both gross pathology and bacteria detected. He concluded that gross pathological changes and intrauterine bacteria were found in $34 \%$ and $20 \%$ of cows, respectively, but the correlation between the two was poor. Abalti et al., (2006) recorded abnormalities in 74 of 201 cow genital tracts. Endometritis represented $3.9 \%$ of the encountered abnormalities. The single case of eosinophilic endometritis appeared to be the result of improper use of the intrauterine antiseptic irrigation e.g. lugol's iodine leading to local allergic reaction.

In women, the high frequency of salpingitis with endometriosis (adenomyosis) may be related to the mechanism of endometriosisassociated infertility (Seidman et al., 2002). Schmeisser et al., (1996) found $86.9 \%$ patent tubes in 130 sterile women underwent first look laparoscopy. In this group, in $34.5 \%$ an endometriosis was discovered. In cases of pelvic endometriosis, Matsushima et al., (2002) found a large number of macrophages using an autosmear method for the tubal collected cells. The etiology of endometriosis and uterine leiomyomata are related to the presence of an estrogen -secreting neoplasm and that the presence of a state of hyperestrogenemia due to granulosa cell tumor over several years might have stimulated the development the endometriosis and leiomyomata (Kurioka et al., 1998). Ferrara et al., (1993) described, in a patient affected by an ovarian granulosa cell tumor (adult type), a case of non-cicatrical endometriosis of the thoracic skin. In cows, adenomyosis is observed occasionally as part of the local disarray of segmental aplasia. It may also be present as a malformation of the tips of the uterine horns in cows (Jubb et al., 1993).

Adenomyosis of the uterine tube is determined by the presence of tubular to cystic components of epithelium of the uterine tube in tunica muscularis and/or similar foci of epithelium of the uterine tube within the subserosa. Tubal adenomyosis was recognized in women as a condition not associated with endometrial adenomyosis (Wrorkand Brooders, 1942; Punnonen et al., 1984). The manner in which it develops has not been resolved. Zhang et al., (1995) examined, pathologically, uterine tubes from 33 women with proximal tubal occlusive infertility. They found tubal endometriosis in $9.80 \%$ of the cases. This condition was also reported in the porcine uterine tube (Kuttel, 1935) and in the bitch and 
queen (McEntee, 1990). Adenomyosis was seen in the cow (Deeb et al., unpublished data). Tomlinson (1998) reported tubal endometriosis in $45 / 405$ cases $(11.1 \%)$ and the majority, 34 (75.6\%) involved the isthmus.

Microscopically, Wordinger and Morrill (1985) examined adult mouse oviduct and endometrium following a single prenatal exposure to diethylstilboesterol. The endometrial changes included squamous metaplasia of both surface and glandular epithelial layer as well as extensive glandular hyperplasia. It was reported that excessive estrogenic stimulation induced endometrial hyperplasia, hydrometra, and mucometra (Jubb et al., 1993). McEntee, (1990) informed that endogenous sources of of estrogen are follicular cysts and granulosa cell tumors, the exogenous sources include synthetic estrogens used for therapeutic purposes and clover pastures containing estrogenic compounds. In the present study, one case of endometrial hyperplasia out of 4 cases was associated with granulose cell tumor. In our opinion, endometrial hyperplasia in these cases may be resulted from estrogenic stimulation of cystic ovarian follicles and granulosa cell tumor as reported by some authors.

Inflammation of the fallopian tubes without significant enlargement is the commonest and most tubal lesion, is usually bilateral, and is usually not detectable macroscopically.

Inflammation in the fallopian tube may occur via hematogenous spread of infectious agents, or by infection ascending from the lower reproductive tract, or descending from the peritoneal cavity. Uterotubal abnormalities, including ovario-tubal adhesions, acute or chronic salpingitis, and hydrosalpinex are said to be more prevalent in the cow than the ewe (McEntee, 1990). Many studies described fallopian tube abnormalities in cattle, the majority reporting a prevalence of $9-19 \%$ using a combination of gross and microscopic observations (Kuttel, 1935; Rowson, 1942; Cembrowicz, 1950, 1956). Duchateau and Whitmore, (1978) found one-half of the occluded bovine uterine tubes (fallopian tubes) (24 cases) were not associated with gross lesions and one half of the tubes with gross lesions were patent. Kessy and Noakes, (1985) recorded tubal lesions in 180/2000 bovine tracts $(9.0 \%) .22$ of the uterine tubes were found to be occluded eventhough they were macroscopically normal. The high frequency of salpingitis in women with endometriosis may be related to the mechanism of endometriosis-associated infertility (Seidman et al., 2002).

However when studies are critically evaluated, there is large variation in the proportion of ovine fallopian tubes reported with abnormalities. The range of lesions observed by macroscopic evaluation is $0.14 \%$ (Emady et al., 1975 ) to $2.70 \%$ (Adams, 1975). With the aid of micrscopic evaluation, there exists a larger variation, from $<1.0 \%(0.8 \%$ for salpingitis and $0.2 \%$ for pyosalpinx (Ahmed et al., 1987) to 7.0\% (Winter and Dobson, 1992), 8.5\% (Rawal et al., (1987), 10.0\% (Sokkar and Kubba (1980), and $11.9 \%$ (Adams, 1975). Gonzalez et al., (1985) reported 16/98 bovine falopian tubes (16.3\%) showing salpingitis. McDougall (2005) isolated bacteria from the uteri of $22(21 \%)$ cows. Isolates included Arcanobacterium pyogens $(\mathrm{n}=1)$, Haemophilus somnus $(\mathrm{n}=5)$, E.coli $(n=1), \quad$ Fusobacterium spp. $(n=1)$, Streptococcus acidominimus $(\mathrm{n}=12)$, S.bovis $(n=2)$, S.uberis $(n=1)$ and S.salivarious $(n=1)$. Gross pathological changes and intrauterine bacteria were found in $34 \%$ and $20 \%$ of cows, respectively, but the correlation between the two was poor.

In an experiment done by Doig et al., (1980), a number of 23 virgin Holstein heifers received uterine inoculations with ureaplasma and were necropsied one to thirteen days later while three heifers inoculated intracervically were necropsied on days 3, 5 and 11 . On histopathology $50 \%$ of uterine inoculated heifers had endometritis upto six days postinoculation and a slightly higher percentage $(58 \%)$ had salpingitis. Salpingitis was not found in any of the three cervical inoculated animals.

According to our results, the picture of the fallopian tube mimics that of the uterus reflecting inflammatory and hormonal changes and occurred most commonly bilaterally. Other abnormalities, e.g., developmental or neoplastic may individually involve the fallopian tube of one or the other side. Pyometra and severe suppurative endometritis were found to be associated with obliteration of the tubal lumen and hence are probable cause of infertility.

\section{Acknowledgement}

Great thanks to Prof. Dr. Salah Deeb Ali for the revision of the manuscript.

\section{References}

Abalti A.; Bekana, M.; Woldemeskel, M. and Lobago, F. (2006): Female genital tract abnormalities of Zebu cattle slaughtered at Bahir-DarTown, north-west Ethiopia. Trop. Animal Health Prod., 38(6):505-510.

Abe, H. and Oikawa, T. (1993): Effects of estradiol and 
progesterone on the cytodifferentiation of the epithelial cells in the oviduct of the newborn golden hamster. Anat Rec., 235(3):390-398.

Bancroft, J. D. and Stevens, A. (1996) Theory and Practice of Histological Techniques Churchill Livingstone, New York. $6^{\text {th }} . e d$

Doig, P. A.; Ruhnke, H. L. and Palmer, N. C. (1980): Experimental bovine genital ureaplasmosis.II. Granular vulvitis, endometritis and salpingitis following uterine inoculation. Can. J. Comp. Med., 44(3):259-266.

Duchateau, A. B.; Whitmore, H. L. (1978): Uterine tube abnormalities in cattle. J. Am. Vet. Med. Assoc., 172 (11): 1308-1309.

Ferrara, G.; Giordano, G.; Longo, M. and Magri, M. (1993): Primary cutaneous endometriosis of thoracic skin with ovarian granulosa cell tumor. Acta Obest. Gynecol. Scand., 72(3):225-227.

Gonzalez, H. E.; Crowell, W. A.; Caudle, A. B., and Thompson, F. N. (1985): Morphometric studies of the bovine uterus: microscopic lesions and retrospective reproductive history. Am. J. Vet. Res., 46(12):2588-2595.

Hatipoglu, F.; Ortatatli, M.; Kiran, M. M.; Erer, H. and Ciftci, M. K. (2002): An abattoir study of genital pathology in cows: II.uterus, cervix and vagina. Revue Med.Vet., 153(2):93-100.

Kessy, B. M. and Noakes, D. E. (1985): uterine tube abnormalities as a cause of bovine infertility. Vet Rec., 117(6):122-124.

Kurioka, H.; Takahashi, K.; Ueda, T.; Ozaki, T. and Miyazaki, K. (1998): Endometriosis and uterine leiomyomata with ovarian granulose cell tumor. Hum Reprod., 13(5):1357-1360.

Matsushima, T.; Kaseki, H.; Ishihara, K. and Araki, T. (2002): Assessment of fallopian tube cytology for the diagnosis of endometriosis and hydrosalpinx. J. Nippon
Med. Sci., 69(5):445-450.

McDougall, S. (2005): Gross abnormalities, bacteriology and histological lesions of uteri of dairy cows failing to conceive or maintain pregnancy. N. Z. Vet. J., 53(4):253256.

Punnonen, R.; Soderstrom, K. O. and Alanen, A. (1984): Isthmic tubal occlusion:etiology and histology. Acta Eur. Fertil., 15(1):39-42.

Schmeisser, J. O.; Krause, T.; Lober, R. and Kohler, G. (1996): Correlation between endometriosis and fallopian tube patenc in sterility patients especially after microsurgical sterility operations. Zentralbl. Gynakol., 118(7):397-400.

Seidman, J. D.; Sherman, M. E.; Bell, K. A.; Katabuchi, H.; O'Leary, T. J. and Kurman, R. J. (2002): Salpingitis,salpingoliths, and serous tumors of the ovaries:is there is a connection? Int. J. Gynecol. Pathol., 21(2):101107.

Tomlinson, L. (1998): Pathology of the ovine female reproductive tract, in particular the uterine tube, and its association with Chlamydia Psittaci.Ph thesis.National library of Canada.395 Wellington street Ottawa ON K1A ON4, Canada.

Verhage, H. G.; Mavrogianis, P. A.; Boice, M. L.; Li, W. and Fazleabas, A. T. (1990): Oviductal epithelium of baboon:hormonal control immuno-gold localization of oviduct-specific glycoproteins. Am. J. Anat., 187(1):8190.

Wordinger, R. J. and Morrill, A. (1985): Histology of the adult mouse oviduct and endometrium following a single prenatal exposure to diethylstilbestrol.Virchows Arch B Cell Pathol. Incl. Mol Pathol., 50(1):71-79.

Zhang, D.; Zeng, Y. and Chen, X. (1995): Pathological findings of proximal tubal occlusive infertility.Zhonghua $\mathrm{Fu}$ Chan Ke Za Zhi., 30(6):352-355.

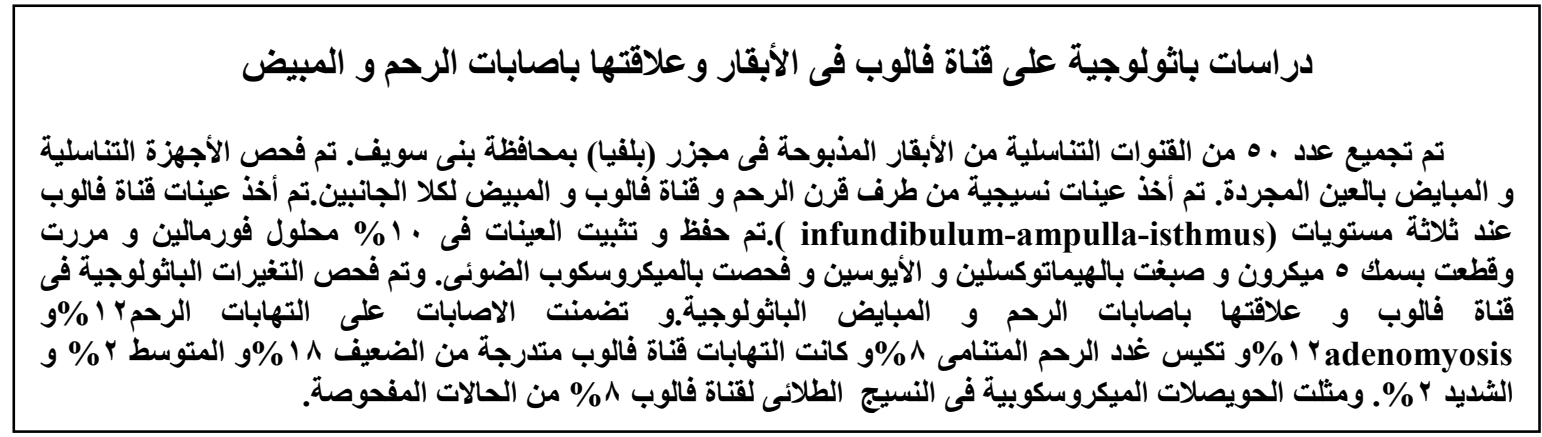

